\title{
Utilização do Digital Smile Design para a correção da estética vermelha do sorriso com técnica cirúrgica suficientemente invasiva
}

\author{
Use of Digital Smile Design for the correction of red smile aesthetics with a sufficiently \\ invasive surgical technique
}

\section{Uso de Digital Smile Design para corregir la estética de la sonrisa roja con una técnica quirúrgica suficientemente invasiva}

Itamar Silva Nunes ${ }^{1 *}$, Júlia Tavares Palmeira1 ${ }^{1}$ Vitor Nascimento Goes ${ }^{1}$, Nilvia Maria Lima Gomes ${ }^{1}$, Laíse da Luz Ramos ${ }^{1}$, José Henrique de Araújo Cruz ${ }^{1}$, Maria Vitória Calado Ramalho dos Santos ${ }^{1}$, Rachel de Queiroz Ferreira Rodrigues ${ }^{1}$, João Nilton Lopes de Sousa' ${ }^{1}$.

\section{RESUMO}

Objetivo: relatar um caso clínico de correção da estética vermelha do sorriso com cirurgia plástica periodontal planejada com auxílio do digital smile design (DSD). Detalhamento do caso: Paciente J.G.G.L. 25 anos de idade, sexo feminino, leucoderma, sistemicamente saudável compareceu a clínica escola de odontologia insatisfeita com seu sorriso. Ao exame clínico periodontal, observou-se que a paciente apresentava sorriso gengival de aproximadamente $4 \mathrm{~mm}$ e coroas clínicas curtas. Foi realizado o protocolo fotográfico necessário ao planejamento digital do sorriso, a fim de verificar por meio da gengivectomia, a quantidade necessária para aumentar as coroas clínicas de cada elemento. A técnica cirúrgica indicada para o caso foi gengivectomia com osteotomia suficientemente invasiva, ou seja, sem levantamento de retalho nos incisivos e caninos superiores. Após 120 dias do procedimento cirúrgico, os resultados revelaram aumento significativo das coroas clínicas e redução do sorriso gengival em $3 \mathrm{~mm}$. Considerações finais: A cirurgia com osteotomia suficientemente invasiva, planejada com o auxilio do DSD, apresenta previsibilidade do resultado e aumenta o sucesso do procedimento cirúrgico periodontal.

Palavras-chaves: Gengiva, Gengivectomia, Estética dentária.

\begin{abstract}
Objective: to report a clinical case of correction of the red smile aesthetics with sufficiently invasive periodontal plastic with the aid of digital smile design (DSD). Case detailing: Patient J.G.G.L. 25-year-old female, leucoderma, systemically healthy attended the clinical school of dentistry unsatisfied with her smile. The periodontal clinical examination revealed that the patient presented approximately $4 \mathrm{~mm}$ of gingival smile and short clinical crowns. The necessary photographic protocol for the digital smile planning was performed in order to verify, through gingivectomy, the amount necessary to increase the clinical crowns of each element. The surgical technique indicated for the case was gingivectomy with osteotomy sufficiently invasive, that is, without lifting the flap in the incisors and upper canines. After 120 days of the surgical procedure, the results revealed a significant increase of the clinical crowns and reduction of the gingival smile in $3 \mathrm{~mm}$. Final considerations: Surgery with a sufficiently invasive osteotomy, planned with the aid of DSD, presents predictability of the result and increases the success of the periodontal surgical procedure.
\end{abstract}

Key words: Gingiva, Gingivectomy, Dental aesthetics.

${ }^{1}$ Universidade Federal de Campina Grande (UFCG). Patos - PB. *E-mail: itamarodontoufcg@gmail.com

SUBMETIDO EM: 2/2020

ACEITO EM: 3/2020

PUBLICADO EM: 4/2020

REAS/EJCH | Vol.Sup.n.47 | e3127 | DOI: https://doi.org/10.25248/reas.e3127.2020 Página 1 de 9 


\section{RESUMEN}

Objetivo: informar un caso clínico de corrección estética de sonrisa roja con cirugía plástica periodontal planificada con la ayuda de sonrisas digitales (dsd). Detalles del caso: paciente J.G.G.L. 25 años, mujer, leucoderma, sistémicamente saludable asistió a la clínica de la escuela dental insatisfecha con su sonrisa. Tras el examen periodontal clínico, se observó que el paciente tenía una sonrisa gingival de aproximadamente $4 \mathrm{~mm}$ y coronas clínicas cortas. El protocolo fotográfico necesario para la planificación de la sonrisa digital se realizó para verificar, a través de la gingivectomía, la cantidad necesaria para aumentar las coronas clínicas de cada elemento. La técnica quirúrgica indicada para el caso fue la gingivectomía con osteotomía lo suficientemente invasiva, es decir, sin levantar colgajos en los incisivos superiores y los caninos. Después de 120 días del procedimiento quirúrgico, los resultados revelaron un aumento significativo en las coronas clínicas y una reducción de $3 \mathrm{~mm}$ en la sonrisa gingival. Consideraciones finales: la cirugía con osteotomía suficientemente invasiva, planificada con la ayuda de DSD, presenta previsibilidad del resultado y aumenta el éxito del procedimiento quirúrgico periodontal.

Palabras clave: Encía, Gingivectomía, Estética dental.

\section{INTRODUÇÃO}

A busca por procedimentos estéticos periodontais, especialmente aqueles que envolvem tratamentos menos invasivos, têm se tornado constante na prática clínica odontológica, principalmente em casos de correções do sorriso gengival (PIRES CV, et al., 2010). Nesse contexto, para a correção dos defeitos mucogengivais que afetam a estética vermelha do sorriso, várias técnicas foram desenvolvidas (CALIXTO $\mathrm{RL}$, et al., 2010). Dentre elas, podem-se citar a gengivectomia, associada ou não à gengivoplastia, a recuperação de espaço biológico e técnicas de aumento de coroa clínica estético suficientemente invasivas (PEDRON IG, et al., 2010).

Dentre as técnicas citadas acima, as cirurgias minimamente invasivas permitem obtenção dos mesmos resultados que a cirurgias periodontais convencionais através de cirurgias substancialmente menores com menor manipulação dos tecidos, e consequentemente menores morbidades para o paciente (PONTES AS, et al., 2016). Para se alcançar bons resultados estéticos na correção do sorriso gengival, é indispensável à avaliação de alguns parâmetros clínicos, tais como: sorriso, lábios, dentes e biótipo periodontal, associados a um bom planejamento cirúrgico para garantir a previsibilidade dos resultados (ZWEERS J, et al., 2014).

Com a evolução tecnológica na odontologia, dispositivos desenvolvidos, como o Computer-Aided Design (CAD-CAM), a modelagem 3D e scanners intra-orais, melhoraram a previsibilidade e precisão dos procedimentos cirúrgicos (STANLEY M, et al., 2018). Neste aspecto, o Digital Smile Design (DSD) tornou-se um método vantajoso para a correção da estética vermelha do sorriso, principalmente no que diz respeito à comunicação entre a equipe, especialista e o paciente (SOUSA JNL, et al., 2019).

Assim o DSD é uma ferramenta que foi desenvolvida com a finalidade de avaliar os parâmetros estéticos, dentais e gengivais, através da análise do registro fotográfico permitindo a avaliação precisa dos resultados desejados em cada fase do tratamento clínico (COACHMAN C, et al., 2012). Nesse contexto, ainda permite e fortalece a capacidade de diagnóstico levando em consideração as características físicas e a expectativa do paciente quanto ao resultado final do tratamento dental (PINTO DCS, et al., 2014).

Dessa forma, o objetivo deste trabalho foi relatar um caso de correção da estética do sorriso por meio de cirurgia plástica periodontal suficientemente invasiva utilizando o DSD como método de diagnóstico e de planejamento pré-operatório.

\section{RELATO DO CASO}

Paciente do gênero feminino, J.G.G.L. 25 anos de idade, Leucoderma, sistemicamente saudável, apresentou-se à Clínica-Escola de Odontologia da UFCG insatisfeita com seu sorriso. Ao exame clínico, foram 
constatados grande exposição gengival e dentes com proporção largura $\mathrm{x}$ altura inadequadas, característicos de um caso de erupção passiva. Após a avaliação periodontal, observou-se ausência de perda de inserção, biótipo periodontal espesso com larga faixa de mucosa queratinizada e linha cervical assimétrica (linha dos zênites gengivais). Os elementos dentários 12, 13, 22 e 23 se encontravam na mesma altura, o zênite do elemento 11 estava posicionado mais para a região incisal quando comparado ao dente 21 (Figura 1).

Figura 1 - Aspectos estéticos do sorriso da paciente.
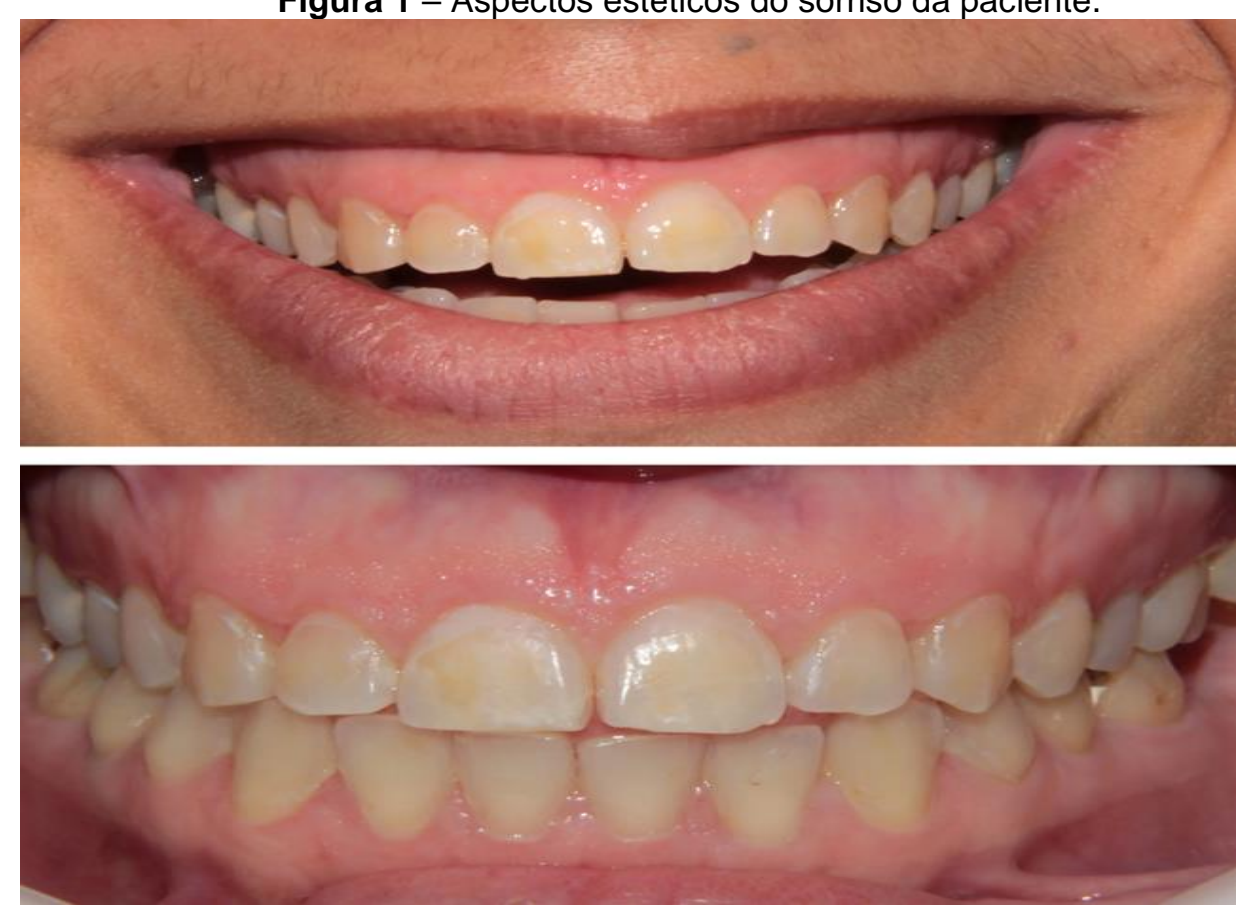

Fonte: Nunes IS, et al., 2020.

Este caso clínico faz parte de um projeto maior não financiado e foi submetido ao Comitê de Ética em Pesquisa das Faculdades Integradas de Patos-PB (CAAE: 59592016.8.0000.5181). O plano de tratamento foi explicado para a paciente, a qual assinou um Termo de Consentimento Livre Esclarecido (TCLE), autorizando a realização do procedimento cirúrgico e publicação deste caso clínico.

Foram realizados exames radiográficos e protocolo fotográfico para a confecção do Digital Smile Design (DSD), ou seja, o planejamento digital do caso na proporção dentária ideal comprimento / largura de $80 \%$ (Figura 2).

Figura 2 - Planejamento DSD: desenho das linhas de referências estéticas e contorno dos dentes guiado pela proporção do retângulo (A) e visão virtual da proporção ideal dos dentes (B e C).

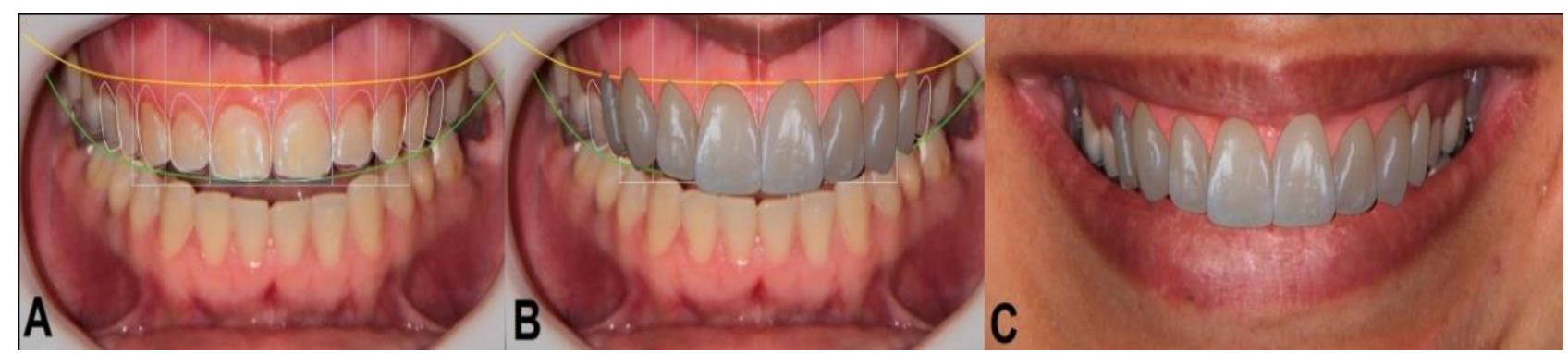

Fonte: Nunes IS, et al., 2020.

Por meio do exame periodontal e das fotografias, observaram-se exposição gengival maior que 3,0 mm, caracterizando sorriso gengival, e os elementos anterossuperiores com proporção dentária estética 
inadequada. As coroas clínicas foram medidas com auxílio de um compasso de ponta seca e uma régua milimétrica. O procedimento cirúrgico escolhido para aumentar as coroas clínicas dos dentes 13 a 23 foi a gengivectomia com osteotomia suficientemente invasiva sem rebatimento de retalho, seguindo os dados do planejamento digital do sorriso. No procedimento cirúrgico, realizou-se antissepsia extra e intrabucal com digluconato de clorexidina 2\% e 0,12\%, respectivamente. Em seguida, anestesia com Articaína $2 \%$ com vasoconstritor, utilizando a técnica infiltrativa no fundo de sulco vestibular e nas papilas vestibulares e palatinas. Com uma sonda periodontal do tipo Williams, foi realizada a marcação, por meio de sondagem transgengival, da faixa de gengiva a ser removida na gengivectomia (Figura 3, A - D).

Figura 3 - Avaliação e marcação da faixa de tecido gengival a ser removido na gengivectomia: A profundidade do sulco gengival, $\mathbf{B}$ - marcação da externa; $\mathbf{C}$ - sondagem transgengival e $\mathbf{D}$ - marcação dos futuros zênites gengivais.
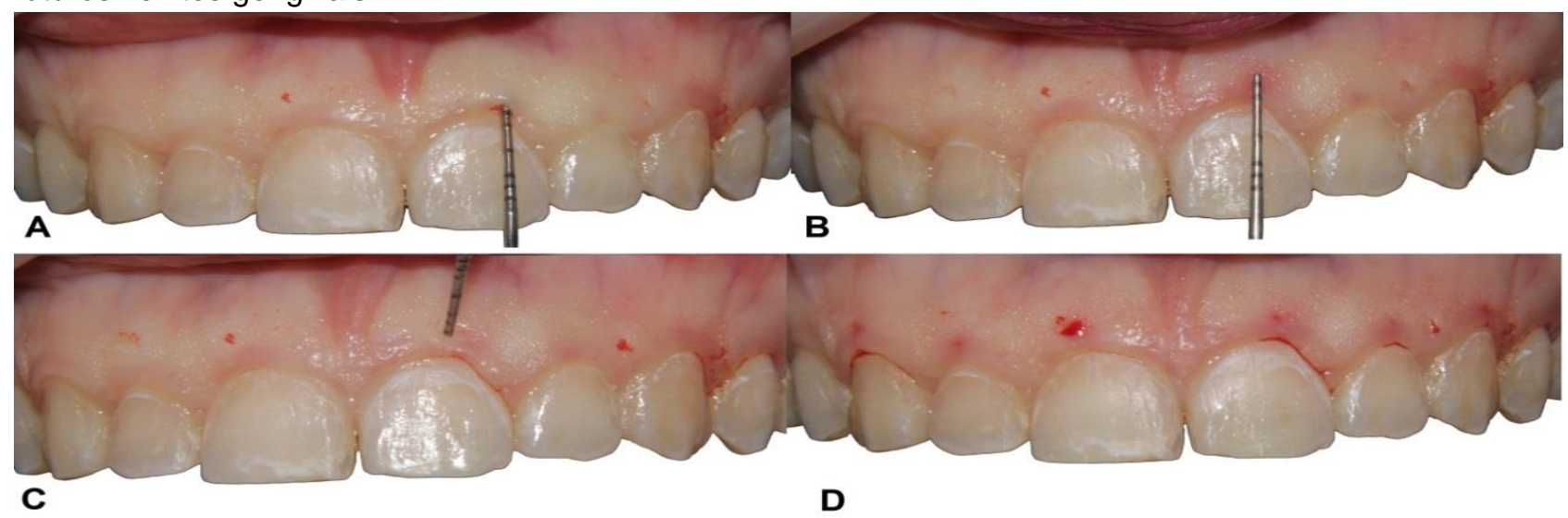

Fonte: Nunes IS, et al., 2020.

A partir de cada ponto marcado, na altura do futuro zênite gengival, foi planejado o contorno da linha de incisão com a ponta da lâmina de bisturi $15 \mathrm{C}$, penetrando superficialmente no tecido gengival e formando uma linha fina visualizada devido ao sangramento. Para um contorno adequado, alinhou-se o zênite do canino para distal. As incisões para remoção do excesso gengival foram realizadas com uma lâmina de bisturi $15 \mathrm{c}$ posicionada a $90^{\circ} \mathrm{em}$ relação ao tecido periodontal. Imediatamente após as incisões, o tecido gengival foi removido com auxílio de uma cureta periodontal de Gracey 5-6. A margem gengival cirúrgica foi posicionada ao nível a junção cemento-esmalte (Figura 4 A e B).

Figura 4 - Gengivectomia e osteotomia:A - remoção do tecido gengival incisado; B - coroas clínicas imediatamente após a gengivectomia; C - avalição do espaço biológico e D - osteotomia via sulco gengival cirúrgico.

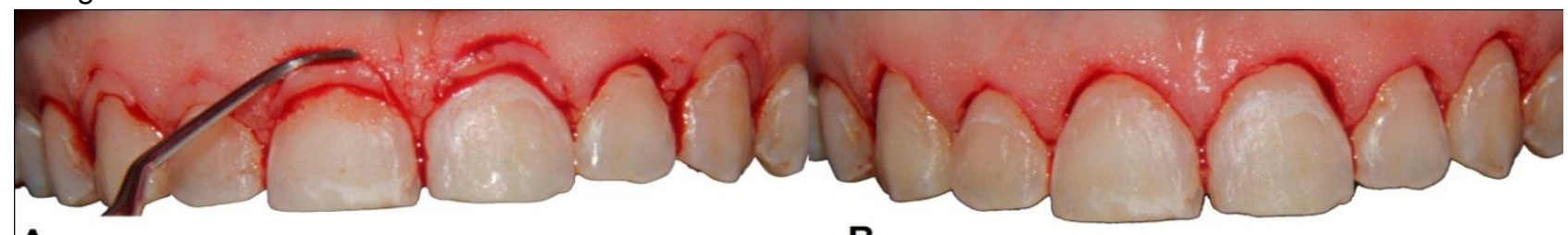

A

B

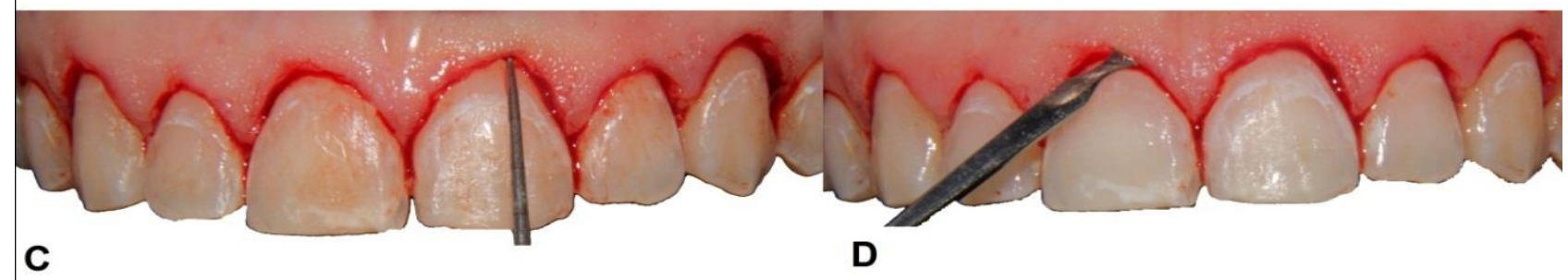

Fonte: Nunes IS, et al., 2020. 
Ao remover o tecido gengival incisado, uma sonda periodontal do tipo WHO OMS foi utilizada para localizar a junção cemento esmalte (JCE) e avaliar a sua distância à crista óssea alveolar (COA). Em seguida foi realizada osteotomia, via sulco gengival cirúrgico, com Cinzel Mini-Ochsenbein №2. Sua lamina ativa foi posicionada a uma angulação de aproximadamente $5^{\circ} \mathrm{em}$ relação ao longo eixo do dente (Figura 4 C e D).

Movimentos leves apicais e de rotação foram realizados no cinzel para remover osso alveolar, distanciando-a em $3 \mathrm{~mm}$ a crista óssea alveolar da junção cemento-esmalte (COA- JCE), aumentando as coroas clínicas dos elementos e restabelecendo as distâncias biológicas periodontais. Após esta etapa, realizou-se remoção dos fragmentos ósseos, raspagem e alisamento em $1 \mathrm{~mm}$ subgengival da superfície radicular e irrigação copiosa com soro fisiológico. Após a osteotomia e osteoplastia, foi realizada irrigação abundante com solução salina a 0,9 \% e não realizou sutura. Em seguida, a paciente foi orientada com relação às instruções de higiene oral e cuidados pós-operatórios. Foi prescrito Dipirona $500 \mathrm{mg}$ apenas em caso de dor.

No pós-operatório de 30 dias, a paciente apresentava um quadro de saúde periodontal, ausência de sangramento gengival, contornos gengivais simétricos, com aspecto de casca de laranja. O resultado do aumento das coroas clínicas e da redução do sorriso gengival da paciente, em relação ao planejamento inicial com o Digital Smile Design (DSD), pode ser observado no (Tabela 1).

Tabela 1 - Planejamento cirúrgico periodontal para avaliar a quantidade de tecido gengival ser removido na gengivectomia e resultado do aumento de coroas clinicas em relação ao planejamento Digital Smile Design.

\begin{tabular}{|c|c|c|c|c|c|}
\hline \multirow[b]{2}{*}{$\begin{array}{l}\text { Elementos } \\
\text { Dentários }\end{array}$} & \multicolumn{2}{|c|}{ Avaliação clínica e periodontal } & \multicolumn{3}{|c|}{ Planejamento no pré-operatório com DSD } \\
\hline & $\begin{array}{l}\text { Coroa clínica } \\
\qquad(\mathrm{mm})\end{array}$ & $\begin{array}{l}\text { Sorriso } \\
\text { gengival }\end{array}$ & \multicolumn{2}{|c|}{$\begin{array}{l}\text { Gengivectomia } \\
\text { (mm) }\end{array}$} & $\begin{array}{l}\text { Coroa clínica } \\
(\mathrm{mm})\end{array}$ \\
\hline 13 & 7,5 & 4 & \multicolumn{2}{|r|}{3} & 10,5 \\
\hline 12 & 6,5 & 4 & \multicolumn{2}{|r|}{2,5} & 9 \\
\hline 11 & 8 & 4 & \multicolumn{2}{|r|}{2,5} & 11,5 \\
\hline 21 & 8 & 3 & \multicolumn{2}{|r|}{2,5} & 11,5 \\
\hline 22 & 7 & 5 & \multicolumn{2}{|r|}{2} & 9 \\
\hline 23 & 7,5 & 4 & \multicolumn{2}{|r|}{3} & 11,5 \\
\hline & \multicolumn{3}{|c|}{ Planejamento no pré-operatório com DSD } & \multicolumn{2}{|c|}{$\begin{array}{l}\text { Resultado do aumento da coroa } \\
\text { clínica após } 30 \text { dias da cirurgia }\end{array}$} \\
\hline $\begin{array}{l}\text { Elementos } \\
\text { Dentários }\end{array}$ & $\begin{array}{l}\text { Coroa clínica } \\
\qquad(\mathrm{mm})\end{array}$ & \multicolumn{2}{|r|}{$\begin{array}{l}\text { Sorriso } \\
\text { gengival } \\
(\mathrm{mm})\end{array}$} & Cor & $\begin{array}{l}\text { Sorriso } \\
\text { gengival }\end{array}$ \\
\hline 13 & 10,5 & & 4 & & 1,5 \\
\hline 12 & 9 & & 4 & & 2,5 \\
\hline 11 & 11,5 & & 4 & & 1 \\
\hline 21 & 11,5 & & 3 & & 0 \\
\hline 22 & 9 & & 5 & & 3 \\
\hline 23 & 11,5 & & 4 & & 0,5 \\
\hline
\end{tabular}

Legenda: DSD - Digital Smile Design

Fonte: Nunes IS, et al., 2020.

No pós-operatório de 120 dias, observou-se que a paciente apresentou um sorriso mais agradável e harmônico (Figura 5) e a mesma relatou melhora de sua autoestima. 


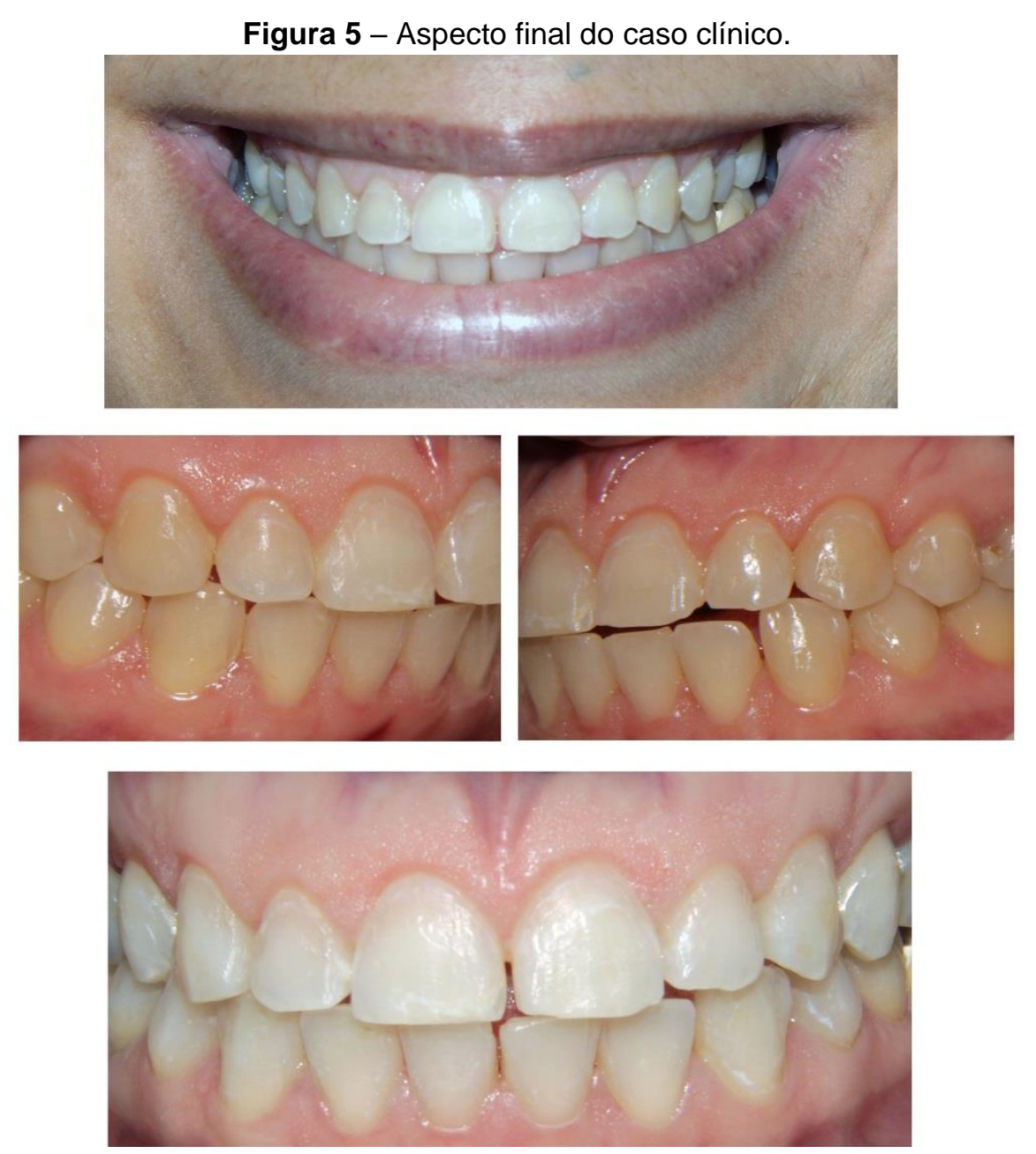

Fonte: Nunes IS, et al., 2020.

\section{DISCUSSÃO}

A necessidade de se obter um sorriso estético aumenta a procura pela cirurgia periodontal (ALVES NV, et al, 2016), porém falhas no equilíbrio entre perfil facial, arquitetura gengival, lábios e dentes ou ainda na estética vermelha (tecido gengival) e no biótipo periodontal são fatores contribuintes neste processo de desarmonia do sorriso (COLDATO KMB, et al., 2018).

Neste aspecto, a literatura define um sorriso aceitável aquele que expõe apenas $1 \mathrm{~mm}$ de tecido gengival muito embora exposições maiores, de até $3 \mathrm{~mm}$ ainda sejam considerados normais (DARAKH P, et al., 2017). Por outro lado, do ponto de vista estético, o sorriso ideal é aquele que apresenta $1 \mathrm{~mm}$ e não mais que $2 \mathrm{~mm}$ de tecido gengival (ZUHR O, et al., 2013). Esse excesso de gengiva promove o desequilíbrio na proporção altura x largura das coroas clínicas bem como, afetam o equilíbrio do posicionamento da linha dos zênites gengivais (FARIA GJ, et al., 2015).

O desequilíbrio na estética do sorriso está associado em grande parte a erupção passiva alterada, caracterizada por uma falha no processo de erupção do dente pelo excesso de gengiva sobre a coroa clínica, que se exibe clinicamente com coroas reduzidas (CAIRO F, et al., 2012), presença de excesso vertical de maxila, onde o problema não é apenas dentário ou no processo eruptivo, mas sim também é um problema esquelético. Podendo ser causado ainda pela hipermobilidade do lábio superior que acarreta em uma exposição excessiva de gengiva durante o ato de sorrir (ZUHR O, et al., 2013).

A etiologia do sorriso gengival é diversa, porém um correto diagnóstico é fundamental na escolha do plano de tratamento mais eficaz (FARIA GJ, et al., 2015). Neste caso, a principal queixa da paciente estava relacionada diretamente com seu desconforto estético, devido a sua coroa clínica reduzida e a exposição 
excessiva de gengiva. Dessa forma, a literatura sustenta que o tratamento adequado depende da etiologia e da severidade da maloclusão que causam o desequilíbrio na estética vermelha do sorriso (ALVARENGA DB, et al., 2018).

Neste aspecto, várias ferramentas são utilizadas para facilitar a comunicação entre o paciente e o cirurgião dentista. Dentre os equipamentos utilizados, a literatura sustenta que as tomadas radiográficas bem como 0 registro fotográfico são uteis a todas as especialidades odontológicas (GONÇALVES PE, et al, 2011). Isso se dá porque o sorriso ideal é aquele que exibe uma relação harmônica entre os dentes, o perfil facial e arquitetura gengival (COACHMAN C, et al., 2012).

Diversos mecanismos podem ser utilizados para processar as imagens na odontologia dentre elas 0 Photoshop e o keynot que, são eficazes em sua capacidade de analisar os parâmetros estéticos e dentogengivais, embora não sejam desenvolvidas para a documentação e design do sorriso (OMAR D e DUARTE C, 2017). Já o DSD que além de permitir traçar qualquer tipo de linha sobre a imagem tem sua interface projetada para o uso odontológico e requer a utilização de programas simples como PowerPoint para analisar imagens de alta qualidade (COACHMAN C, et al., 2012).

Dessa forma, este estudo utilizou o DSD para realizar o planejamento do sorriso mais adequado de acordo com a proporção dentária ideal (comprimento x largura) com base nas características faciais, dentárias e gengivais da paciente, que permitisse atender as suas necessidades estéticas, funcionais e emocionais bem como auxiliar no planejamento cirúrgico. Estando em concordância com a literatura, o planejamento digital deve permitir previsibilidade e satisfação do individuo, como também ser de fácil manuseio (PINTO DCS, et al., 2014).

No que diz respeito ao tratamento da exposição excessiva de gengiva a literatura relata que o tratamento depende da sua etiologia, quando a alteração é causada pelo excesso vertical de maxila o tratamento de escolha é a cirurgia ortognática, quando a desordem é causada pela erupção ativa a opção de escolha é o tratamento ortodôntico, porém quando se trata de um caso de erupção passiva alterada a cirurgia periodontal é o tratamento de primeira escolha (ZUHR O, et al., 2013).

Em relação à cirurgia periodontal a gengivectomia consiste em uma medida eficaz no aumento da coroa clínica e no estabelecimento de um contorno fisiológico mais adequado, onde $73 \%$ dos pacientes submetido a este procedimento apresentam-se satisfeitos com a exposição gengival e dentária após a cirurgia (SILVA $\mathrm{CO}$, et al, 2015). Além disso, esta técnica é importante para o estabelecimento adequado da distância biológica (BRAGA MS, et al., 2015).

No presente caso clínico, optou-se pela gengivectomia com osteotomia suficientemente invasiva e bisel levemente interno, uma vez, que permite o estabelecimento da proporção comprimento/largura desejados pelo planejamento digital de forma menos traumática. Sustentado pela literatura que determinam que procedimentos do tipo flapless reduzem o trauma ao osso adjacente, como também minimizam risco do paciente desenvolverem complicações pós-operatórias (JOB S e BHAT V, 2008).

No que diz respeito à morbidade, a técnica convencional com elevação do retalho gera maior trauma aos tecidos, reduz o suprimento sanguíneo gerando desconforto pós-operatório para o paciente (JOB S e BHAT V, 2008). Por outro lado, a técnica sem elevação do retalho proporciona maior vascularização da área cirúrgica e consequentemente diminui, o processo inflamatório como também minimiza a dor pós-operatória (KIM J, et al., 2008). Estando em concordância com este trabalho uma vez que a paciente relatou não sentir necessidade de fazer uso da medicação pós-operatória prescrita em caso de dor. Neste caso, a gengivectomia com osteotomia suficientemente invasiva realizada via sulco gengival, dispensa a sutura e permite a cicatrização dos tecidos por primeira intenção, devido à preservação da papila e a não realização de incisões relaxantes (DANNAN A, 2011). Assim, a cirurgia atraumática requer menor tempo cirúrgico quando comparado à cirurgia convencional, favorecendo a recuperação e a higienização da cavidade bucal (PONTES AS, et al., 2016).

A principal limitação da técnica está relacionada à necessidade de equipamentos mais delicados como micro-tesouras, micro-cinzéis, micro-lâminas que permitam maior controle sobre as incisões e 
desbridamentos cirúrgicos quando comparados à técnica convencional (CORTELLINI $P, 2012$ ). Outra desvantagem está relacionada ao acesso, que é cego via sulco gengival guiado por sonda que não permite a visualização direta dos tecidos subjacentes (PONTES AS, et al., 2016).

A técnica convencional, por sua vez, é eficaz no aumento de coroa clínica com osteoplástia em casos de um biótipo periodontal espesso tipo I, em que há a presença de uma exostose significante (BORGES GS, et al, 2012). O acesso amplo é fator determinante para permitir a visualização direta do tecido ósseo objetivando permitir a regularização em espessura do rebordo alveolar (SILVA DB, et al., 2010). Desta forma este trabalho ainda está em concordância com Trevisani RS e Von Meusel DRDZ (2015) que defende que, apesar das limitações da cirurgia minimamente invasiva, está é uma medida útil na correção da estética vermelha do sorriso por meio de uma técnica de fácil execução com menor morbidade trans e pós-operatórias por gerar menor trauma ao periodonto e aos tecidos subjacentes.

O DSD é uma ferramenta odontológica desenvolvida por Coachman C, et al. (2012) ao qual, permite a análise dos parâmetros clínicos por meio do registro fotográfico. Sendo eficaz quanto à capacidade de avaliar os parâmetros dentais, gengivais e faciais (PINTO DCS, et al., 2014).

Por sua vez, o DSD é uma ferramenta importante no planejamento clínico, favorecendo o diagnóstico e permitindo uma melhor comunicação entre o cirurgião dentista e o paciente, garantindo a previsibilidade dos resultados esperados por ambos (OMAR D e DUARTE C, 2017). Estando em concordância com este caso clínico onde o planejamento digital permitiu uma melhor aceitação da paciente ao tratamento proposto para a correção da estética vermelha do sorriso.

\section{CONSIDERAÇÕES FINAIS}

A cirurgia de aumento de coroa clínica estético pela técnica suficientemente invasiva planejada com auxílio do desenho digital do sorriso apresenta previsibilidade de resultado e reduz a morbidade do procedimento cirúrgico.

\section{REFERÊNCIAS}

1. ALVARENGA DB, et al. Inter-relação periodontia/dentística na correção de sorriso gengival: relato de caso clínico. Rev. Braz J Periodontol, 2018; 28: 7-13.

2. ALVES NV, et al. Reabilitação estética e funcional do sorriso: revisão de literatura. Rev Inter SaúHumaTec, JuazNor, 2016; 3: 25-30.

3. BORGES GJ, et al. Aumento de coroa estético associado ao reposicionamento labial com cimento ortopédico. RevOdont do BrasCentr, 2012; 21: 57.

4. BRAG MS, et al. Cirurgia plástica periodontal para correção de erupção passiva alterada. Braz J Periodontol, 2015; 25: 04.

5. CAIRO F, et al. Periodontal plastic surgery to improve aesthetics in patients with altered passive eruption/gummy smile: a case series study. Rev. Inter JourDent, 2012; 1-6.

6. CALIXTO RL, et al. Correção de desnível de margem gengival: interação periodentística no restabelecimento do sorriso. Rev. Clínica - International Journal of Brazilian Dentistry, Florianópolis, 2010; 6(4): 434-441.

7. COACHMAN C, CALAMITA M, SCHAYDER A. Digital smile design: uma ferramenta para planejamento e comunicação em odontologia estética. Rev. Dicas, 2012; 1: 36-55.

8. COLDATO KMB, et al. Avaliação do biotipo periodontal em áreas acometidas por recessão gengival em acadêmicos de odontologia. Rev. Braz J Periodontol, 2018; 28: 19-27.

9. CORTELLINI P. Minimally Invasive Surgical Techniques in Periodontal regeneration. Rev. J Evid Base Dent Pract, 2012; 12(1): 89-100.

10. DANNAN A. Minimally invasive periodontal therapy. Rev. Article, 2011; 15(4): 338-343.

11. DARAKH P, et al. Treatment of altered passive eruption by surgical gingivectomy: A case report. Rev. International Journal of Periodontology and Implantology, 2017; 2: 64-68.

12. FARIA GJ, et al. A importância do planejamento multidiscipilinar para correção do sorriso gengival: relato de caso clínico. Rev da FaculOdontoeLins, 2015; 25: 61-66.

13. GONÇALVES PE, DOTTA EAV, SERRA MC. Imageologia na odontologia e aspectos legais. Rev. Gaúcha Odontol, 2011; 59: 89-95. 
14. JOB S, BHAT V. An insight into flapless implant placement technique. Rev. The Journal of Indian Prosthodontic Society, 2008; 8(3).

15. KIM J, et al. Blood vessels of the peri-implant mucosa: a comparison between flap and flapless procedures. Rev. OOOOE, 2008; 107(4): 508-512.

16. OMAR D, DUARTE C. The application of parameters for comprehensive 5 smile esthetics by digital smile design programs: 6 A review of literature. Rev. The Saudi Dental Journal, 2017; 01-06.

17. PEDRON IG, et al. Sorriso gengival: cirurgia ressectiva coadjuvante à estética dental. Rev. Odonto. São Paulo,2010; 35(18): 87-95. Disponível em: <https://www.metodista.br/revistas/revistasims/index.php/Odonto/article/view/1564>. Acesso em: 21 out. 2017.

18. PINTO DCS, et al. Desenho digital do sorriso-Descrição de uma nova técnica. Rev. Gestão e Saúde, $2014 ; 11$ : 01-09.

19. PIRES CV, et al. Procedimentos plásticos periodontais em paciente com sorriso gengival - relato de caso. Rev. Periodontia, 2010; 20: 01.

20. PONTES AS, et al. Aumento de coroa clínica estético minimamente invasivo: relato de caso de 12 meses. Rev. Saúde, 2016; 10: 3-4.

21. SILVA CO, et al. Aesthetic crown lengthening: periodontal and patient-centred outcomes. Rev. Journal of clinical periodontology, 2015; 42(12): 1126-1134.

22. SILVA DB, et al. Cirurgia plástica periodontal para otimização da harmonia dentogengival - relato de caso clínico. Rev. Braz Jour of Heal, 2010; $1: 31$.

23. SOUSA JNL, et al. Desenho digital do sorriso no planejamento interdisciplinar entre periodontia e prótese - relato de caso. Rev. Prosthes. Esthet. Sci, 2019; 8(30): 55-67.

24. STANLEY M, et al. Fully digital workflow, integrating dental scan, smile design and CAD-CAM: case report. Rev. BMC Oral Health, 2018; 18: 2-8.

25. TREVISANI RS, VON MEUSEL DRDZ. Aumento de coroa clínica em dentes anteriores. Rev. JourofOrInvest, 2015; 3: 19-24.

26. ZUHR O, et al. Aumento de coroa estético. In: Zuhr O, Hurgller. Cirurgia plástica estética, periodontal e implantar. Uma abordagem microcirúrgica. Germany: Quintessence editora Ltda; 2013.

27. ZWEERS J, et al. Characteristics of periodontal biotype, its dimensions, associations and prevalence -a systematic review. Rev. Journal of Clinical Periodontology, 2014: 1-25. 